\title{
Average observability of large-scale network systems
}

\author{
Muhammad Umar B. Niazi, Carlos Canudas-de-Wit and Alain Y. Kibangou
}

\begin{abstract}
This paper addresses observability and detectability of the average state of a network system when few gateway nodes are available. To reduce the complexity of the problem, the system is transformed to a lower dimensional state space by aggregation. The notions of average observability and average detectability are then defined, and the respective necessary and sufficient conditions are provided.
\end{abstract}

Index Terms - Large-scale systems, state aggregation, average observability, average detectability.

\section{INTRODUCTION}

Graph-theoretic approaches for controllability and observability of network systems have been extensively studied in the past few decades, [1]-[6]. A resulting problem of interest has been to identify the minimum number of gateway nodes through which a network system is controllable or, respectively, observable.

In large-scale network systems, however, we face the issues of system complexity and limited sensing capability. Complexity challenges the computational resources at hand and a limited number of sensors may render the network system unobservable. Moreover, knowing the complete state of a network is often unnecessary for control and monitoring purposes. For instance, in state feedback [7], some linear functionals of the state are usually required.

In this paper, we study the observability of an average state of a large-scale network system when few gateway nodes are available. Network nodes where sensors are deployed to obtain dedicated state measurements are called gateway nodes (or measured nodes). The rest of the nodes are called unmeasured nodes. The average state is meaningful in many applications, especially for positive systems [8], where the average provides a suitable estimate of the state norm, which is useful in feedback stabilization [9].

We investigate whether it is possible to reconstruct an average state of a network system from the state measurements at gateway nodes. This befalls under the problem of functional observability, where one is interested in reconstructing a set of linear functionals of the states. However, the necessary and sufficient condition of functional observability in [10], [11] requires the computation of ranks of a concatenation of system matrices, which is not feasible when dealing with

M. U. B. Niazi and C. Canudas-de-Wit are with CNRS, GIPSA-Lab, Grenoble, France. \{muhammad-umar-b.niazi, carlos. canudas-de-wit\}@gipsa-lab.fr

A. Y. Kibangou is with Université Grenoble Alpes, CNRS, Inria, Grenoble INP, GIPSA-Lab, Grenoble, France. alain.kibangouduniv-grenoble-alpes.fr

This work is supported by European Research Council (ERC) under the European Union's Horizon 2020 research and innovation programme (ERCAdG no. 694209, Scale-FreeBack, website: http://scale-freeback.eu/). large-scale systems. Furthermore, we use the term average observability to emphasize that an average is the quantity of interest and not an arbitrary linear functional of the states. Nevertheless, the approach can be generalized for any linear functional of the state.

We present a different approach to examine average observability of a network system by transforming it to a lowerdimensional state space, which is shown to be influenced by a vector of 'deviation' from the average. Thus we provide necessary and sufficient conditions for average observability that are computationally tractable for large-scale networks. In addition, we also provide the conditions of average detectability, which is a notion that concerns with the stability of the average state.

The paper is organized as follows. In Section II, we formulate the problem. In Section III and IV, we define and study the notions of average observability and average detectability, respectively. Finally, in Section V, we present conclusions and future prospects. The technical proofs of the results are deferred to Appendix. We abide by the following notations throughout the paper:

Notations: The set of real and complex numbers are denoted as $\mathbb{R}$ and $\mathbb{C}$, respectively. The sets $\mathbb{C}_{<0}$ and $\mathbb{C}_{\geq 0}$ represent open left-half and closed right-half complex planes, respectively. We differentiate between scalars and vectors by using boldface lowercase letters for vectors. The uppercase letters are reserved for matrices. The identity matrix and vector of ones are denoted as $I_{n} \in \mathbb{R}^{n \times n}$ and $\mathbf{1}_{n} \in \mathbb{R}^{n}$, respectively. The set of eigenvalues of a square matrix $A$ is denoted as $\operatorname{eig}(A) \subset \mathbb{C}$. We denote by $\operatorname{diag}\left[A_{1}, \ldots, A_{k}\right]$ a block diagonal matrix with matrices $A_{1}, \ldots, A_{k}$ at its diagonal.

\section{PROBLEM FORMULATION}

Consider a network represented by a weighted directed graph $\mathcal{G}=(\mathcal{V}, \mathcal{E})$, where $\mathcal{V}=\left\{v_{1}, v_{2}, \ldots, v_{n}\right\}$ is the set of nodes indexed by the set $\mathcal{I}=\{1, \ldots, n\}$ and $\mathcal{E} \subseteq \mathcal{V} \times \mathcal{V}$ is the set of directed edges or arcs. The edge configuration of the nodes is given by the adjacency matrix $W \in \mathbb{R}^{n \times n}$, whose $i j$-th entry is given by

$$
[W]_{i j}= \begin{cases}w_{i j}, & \text { if } i \neq j \text { and }\left(v_{i}, v_{j}\right) \in \mathcal{E} \\ 0, & \text { otherwise }\end{cases}
$$

where $w_{i j}>0$ is the weight of the edge $\left(v_{i}, v_{j}\right) \in \mathcal{E}$. We follow the convention that the edge $\left(v_{i}, v_{j}\right)$ is represented as $v_{i} \leftarrow v_{j}$. Hence, if $\left(v_{i}, v_{j}\right) \in \mathcal{E}$, then we say that there is an information flow (or inflow) to $v_{i}$ from $v_{j}$. The state $x_{i}(t)$ 


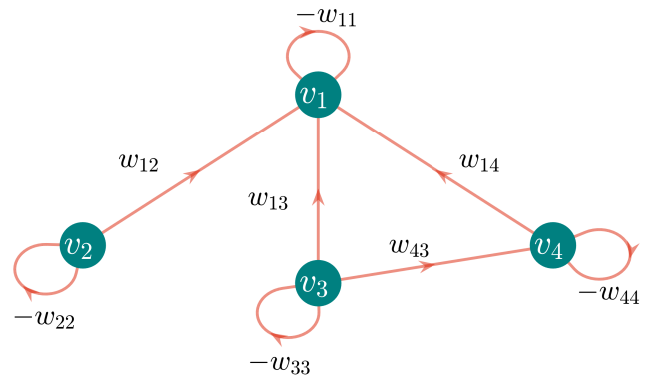

Fig. 1: Network system

of each node $v_{i} \in \mathcal{V}$ satisfies

$$
\dot{x}_{i}(t)=-w_{i i} x_{i}(t)+\sum_{j \in \mathcal{N}_{i}^{i n}} w_{i j} x_{j}(t)+\sum_{l=1}^{p} b_{i l} u_{l}(t),
$$

where $w_{i i} \geq 0$ can be considered as a local-damping weight, $u_{l}(t) \in \mathbb{R}$ is an $l$-th input applied at node $v_{i}$ with a scaling factor $b_{i l} \in \mathbb{R}$, and $\mathcal{N}_{i}^{i n}:=\left\{j \in \mathcal{I}:\left(v_{i}, v_{j}\right) \in \mathcal{E}\right\}$ is the set of indexes of $v_{i}$ 's in-neighbors. Network system (1), as shown in Figure 1, is represented with self-loops at nodes due to local-damping.

Remark 1. We remark that (1) is a general model for linear time-invariant (LTI) network systems, where the value of local damping weight $w_{i i}$ is free. For instance, in a reactiondiffusion system evolving over undirected networks, [12], each node has $w_{i i}=r_{i}+\sum_{j \in \mathcal{N}_{i}^{i n}} w_{i j}$, where $r_{i}>0$ is the reaction rate and $w_{i j}=w_{j i}$ is the diffusion intensity between the nodes $v_{i}$ and $v_{j}$. Similarly, in a multi-agent system seeking consensus, [2], we have $w_{i i}=\sum_{j \in \mathcal{N}_{i}^{i n}} w_{i j}$, or in a linear multi-compartmental system, we have $w_{i i}=$ $\sum_{j \in \mathcal{N}_{i}^{\text {out }}} w_{j i}$, where $\mathcal{N}_{i}^{\text {out }}:=\left\{j \in \mathcal{I}:\left(v_{j}, v_{i}\right) \in \mathcal{E}\right\}$ is the set of indexes of $v_{i}$ 's out-neighbors.

Let $\mathbf{x}(t)=\left[x_{1}(t) \ldots x_{n}(t)\right]^{T}$ be the network state vector and $\mathbf{u}(t)=\left[u_{1}(t) \ldots u_{p}(t)\right]^{T}$ be the input vector, then a linear time-invariant (LTI) network system (1) can be written in a state-space form:

$$
\boldsymbol{\Sigma}:\left\{\begin{array}{l}
\dot{\mathbf{x}}(t)=A \mathbf{x}(t)+B \mathbf{u}(t) \\
\mathbf{y}(t)=C \mathbf{x}(t)
\end{array},\right.
$$

where $A \in \mathbb{R}^{n \times n}, B \in \mathbb{R}^{n \times p}$, and $C \in \mathbb{R}^{n_{1} \times n}$. The output vector $\mathbf{y}(t)$, for $t \geq 0$, contains the dedicated state measurements of $n_{1}<n_{2}$ nodes, where $n_{1}$ is the number of gateway nodes and $n_{2}$ the number of unmeasured nodes with $n_{1}+n_{2}=n$. Note that $A=W-D$, where $D=$ $\operatorname{diag}\left[w_{11}, \ldots, w_{n n}\right]$, and $[B]_{i l}=b_{i l}$ for $i=1, \ldots, n$ and $l=1, \ldots, p$.

\section{A. Preliminaries of observability and detectability}

Considering the LTI systems of the form $\boldsymbol{\Sigma}$, we briefly recall the notions of observability and detectability. Note that the state trajectory is given by

$$
\mathbf{x}(t)=e^{A t} \mathbf{x}(0)+\int_{0}^{t} e^{A(t-\tau)} B \mathbf{u}(\tau) d \tau,
$$

and the output

$$
\mathbf{y}(t)=C e^{A t} \mathbf{x}(0)+\int_{0}^{t} C e^{A(t-\tau)} B \mathbf{u}(\tau) d \tau .
$$

To determine the state trajectory of $\boldsymbol{\Sigma}$, it is necessary and sufficient to know the initial state $\mathbf{x}(0)$. Thus, observability is a property of a system that ensures that the initial state $\mathbf{x}(0)$ can be reconstructed from the knowledge of inputs $\mathbf{u}(t)$ and outputs $\mathbf{y}(t)$ over $t \in[0, \infty)$. It is well-known, [7], that a system represented as $\boldsymbol{\Sigma}$ is observable if and only if the pair $(C, A)$ is observable, that is,

(a) $\operatorname{rank} \mathcal{O}_{C, A}=n$, which is known as observability rank condition and the observability matrix is defined as

$$
\mathcal{O}_{C, A}:=\left[\begin{array}{l}
C \\
C A \\
\vdots \\
C A^{n-1}
\end{array}\right] ;
$$

(b) $\operatorname{rank}\left[\begin{array}{c}s I-A \\ C\end{array}\right]=n$, for all $s \in \operatorname{eig}(A)$, which is known as Popov-Belevitch-Hautus (PBH) test.

The above conditions are equivalent and commonly used to test the observability of an LTI system. If the PBH test fails, i.e., rank $\left[(s I-A)^{T} C^{T}\right]^{T}<n$ for $s \in \mathcal{X} \subseteq \operatorname{eig}(A)$, then $\Sigma$ is said to be detectable if and only if $\operatorname{Re}\{s\}<0$ for every $s \in \mathcal{X}$. That is, $\boldsymbol{\Sigma}$ is detectable if and only if

$$
\operatorname{rank}\left[\begin{array}{c}
s I-A \\
C
\end{array}\right]=n \text {, for all } s \in \mathbb{C}_{\geq 0} .
$$

If the system is not observable, then there are some unobservable modes of the state that cannot be reconstructed. However, if all the unobservable modes are stable, then the system is detectable and one can obtain an asymptotic estimate of the state by an observer [13].

\section{B. Average state of unmeasured nodes}

Due to limited number of available sensors, we assume that $\boldsymbol{\Sigma}$ is not observable. Therefore, we resort to the problem of reconstructing an average state of unmeasured nodes.

Without loss of generality, we suppose the state partition as $\mathbf{x}(t)=\left[\begin{array}{lll}\mathbf{x}_{1}^{T}(t) & \mathbf{x}_{2}^{T}(t)\end{array}\right]^{T}$, where $\mathbf{x}_{1}(t) \in \mathbb{R}^{n_{1}}$ and $\mathbf{x}_{2}(t) \in \mathbb{R}^{n_{2}}$ are the states of gateway nodes and unmeasured nodes, respectively. To obtain this partition, one can simply reorder the network nodes by transforming the network state vector $\mathbf{x}(t)$ with an appropriate permutation matrix. Then, $\mathcal{V}=\mathcal{V}_{1} \cup \mathcal{V}_{2}$, where the subsets $\mathcal{V}_{1}=\left\{v_{1}, \ldots, v_{n_{1}}\right\}$ and $\mathcal{V}_{2}=\mathcal{V} \backslash \mathcal{V}_{1}$ contain the gateway nodes and unmeasured nodes, respectively, and we obtain the following block structure of system matrices in $\Sigma$ :

$$
\begin{aligned}
& A=\left[\begin{array}{ll}
A_{11} & A_{12} \\
A_{21} & A_{22}
\end{array}\right], \quad B=\left[\begin{array}{c}
B_{1} \\
B_{2}
\end{array}\right], \\
& C=\left[\begin{array}{ll}
I_{n_{1}} & 0
\end{array}\right] .
\end{aligned}
$$

Let the average state $\bar{x}(t):=\mathbf{p}^{T} \mathbf{x}_{2}(t)$ be a linear combination of the states of unmeasured nodes, where $\mathbf{p}=\frac{1}{\sqrt{n_{2}}} \mathbf{1}_{n_{2}} \in \mathbb{R}^{n_{2}}$ such that $\mathbf{p}^{T} \mathbf{p}=1$. Note that $\bar{x}(t)$ is 
an average mean scaled by $\sqrt{n_{2}}$. This scaling is merely for the sake of convenience. Let

$$
\boldsymbol{\sigma}(t):=\mathbf{x}_{2}(t)-\mathbf{p} \bar{x}(t)
$$

be a deviation vector and note that $\mathbf{p}^{T} \boldsymbol{\sigma}(t)=0$.

\section{Problem statement}

Given a network system $\Sigma$, under what conditions is it possible to reconstruct the average state $\bar{x}(t)=\mathbf{p}^{T} \mathbf{x}_{2}(t)$ if the knowledge of dedicated state measurements at gateway nodes $\mathbf{y}(t)$ and input $\mathbf{u}(t)$ is available over $t \in[0, \infty)$ ? The problem is concerned with the observability of the average state of unmeasured nodes, which is called average observability. On the other hand, under what conditions the dynamics of the average state is stable? That is, if $\mathbf{x}_{1}(t)=0$ and $\mathbf{u}(t)=0$, then do we have $\bar{x}(t) \rightarrow 0$ as $t \rightarrow \infty$ ? This is called average detectability.

\section{AVERAGE OBSERVABILITY OF NETWORK SYSTEMS}

In this section, we state necessary and sufficient conditions for average observability of network systems by projecting the state of $\boldsymbol{\Sigma}$ to a lower-dimensional state space. Thus, we obtain a projected network system, which is shown to be influenced by a deviation vector $\boldsymbol{\sigma}(t)$.

To derive a model of projected network system, we consider a lower-dimensional projection of the network state,

$$
\mathbf{z}(t)=P \mathbf{x}(t)
$$

where $\mathbf{z}(t)=\left[\begin{array}{ll}\mathbf{x}_{1}^{T}(t) & \bar{x}(t)\end{array}\right]^{T} \in \mathbb{R}^{n_{1}+1}$ and

$$
P=\left[\begin{array}{cc}
I_{n_{1}} & 0 \\
0 & \mathbf{p}^{T}
\end{array}\right], \quad P^{T}=\left[\begin{array}{cc}
I_{n_{1}} & 0 \\
0 & \mathbf{p}
\end{array}\right]
$$

such that $P P^{T}=I$. Note that $\mathbf{x}(t)=P^{T} \mathbf{z}(t)+\mathbf{h}(t)$, where $\mathbf{h}(t)=\left[\begin{array}{ll}\mathbf{0}^{T} & \boldsymbol{\sigma}^{T}(t)\end{array}\right]^{T}$. Thus, we obtain the following system

$$
\boldsymbol{\Sigma}_{P}:\left\{\begin{array}{l}
\dot{\mathbf{z}}(t)=E \mathbf{z}(t)+F \boldsymbol{\sigma}(t)+G \mathbf{u}(t) \\
\mathbf{y}(t)=H \mathbf{z}(t),
\end{array}\right.
$$

where $E=P A P^{T}, F \boldsymbol{\sigma}(t)=P A \mathbf{h}(t), G=P B$, and $H=C P^{T}$;

$$
\begin{aligned}
E & =\left[\begin{array}{cc}
A_{11} & A_{12} \mathbf{p} \\
\mathbf{p}^{T} A_{21} & \mathbf{p}^{T} A_{22} \mathbf{p}
\end{array}\right], \quad F=\left[\begin{array}{c}
A_{12} \\
\mathbf{p}^{T} A_{22}
\end{array}\right], \\
G & =\left[\begin{array}{c}
B_{1} \\
\mathbf{p}^{T} B_{2}
\end{array}\right], \quad H=\left[\begin{array}{ll}
I_{n_{1}} & 0
\end{array}\right] .
\end{aligned}
$$

Notice that the deviation vector $\boldsymbol{\sigma}(t)$ enters the system $\boldsymbol{\Sigma}_{P}$ as an 'unknown' input, because $\boldsymbol{\sigma}(t)=\mathbf{x}_{2}(t)-\mathbf{p} \bar{x}(t)$ and $\mathbf{x}_{2}(t)$ is not measured. However, since $\boldsymbol{\sigma}$ is not an arbitrary disturbance and $\boldsymbol{\Sigma}_{P}$ is the projection of $\boldsymbol{\Sigma}$ on lowerdimensional state space, we consider the observability (resp., detectability) of $\boldsymbol{\Sigma}_{P}$ equivalent to the average observability (resp., average detectability) of $\boldsymbol{\Sigma}$.

Lemma 1. The pair $(H, E)$ in $\boldsymbol{\Sigma}_{P}$ is an observable pair if and only if there exists an edge $(i, j) \in \mathcal{E}$, where $i \in \mathcal{V}_{1}$ is a gateway node and $j \in \mathcal{V}_{2}$ is an unmeasured node.

The observability of the pair $(H, E)$ is a necessary condition for the observability of $\Sigma_{P}-$ it is, however, necessary and sufficient only when $F \boldsymbol{\sigma}(t)=0$ for all $t \geq 0$. Since it requires just one edge (inflow) to the gateway nodes from unmeasured nodes, it is appropriate to make the following assumption:

Assumption 1. There exists at least one edge $(i, j) \in \mathcal{E}$ such that $i \in \mathcal{V}_{1}$ is a gateway node and $j \in \mathcal{V}_{2}$ is an unmeasured node.

We introduce the notion of average observability as the property of $\boldsymbol{\Sigma}$ which ensures the reconstruction of the average state $\bar{x}(t)$ from $\boldsymbol{\Sigma}_{P}$ by assuming the knowledge of state measurements $\mathbf{x}_{1}(t)$ at the gateway nodes and the input $\mathbf{u}(t)$ for all $t \geq 0$. Note that the output $\mathbf{y}(t)$ of the systems $\boldsymbol{\Sigma}$ and $\boldsymbol{\Sigma}_{P}$ is same and is given by

$$
\mathbf{y}_{\boldsymbol{\sigma}}(t, \mathbf{z}(0))=H e^{E t} \mathbf{z}(0)+\int_{0}^{t} H e^{E(t-\tau)}[F \boldsymbol{\sigma}(\tau)+G \mathbf{u}(\tau)] d \tau .
$$

Precisely, average observability is defined as:

Definition 1. Suppose $\mathbf{u}(t)=0$ in $\boldsymbol{\Sigma}$. Let $\bar{x}(t)=\mathbf{p}^{T} \mathbf{x}_{2}(t)$ with $\mathbf{p}=n_{2}^{-\frac{1}{2}} \mathbf{1}_{n_{2}}$. Then, $\boldsymbol{\Sigma}$ is said to be average observable if for all initial conditions $\mathbf{z}(0)=\left[\begin{array}{ll}\mathbf{x}_{1}^{T}(0) & \bar{x}(0)\end{array}\right]^{T} \in \mathbb{R}^{n_{1}+1}$ and the deviation vector $\boldsymbol{\sigma}(t) \in \mathbb{R}^{n_{2}}$ is such that $\mathbf{p}^{T} \boldsymbol{\sigma}(t)=0$ for all $t \geq 0$, it holds that the output $\mathbf{y}_{\boldsymbol{\sigma}}(t, \mathbf{z}(0))=\mathbf{x}_{1}(t)=0$ for all $t \geq 0$ implies $\bar{x}(0)=0$, where $\mathbf{y}_{\boldsymbol{\sigma}}(t, \mathbf{z}(0))$ is given in (6).

If the initial average $\bar{x}(0) \neq 0$ but $\mathbf{y}_{\boldsymbol{\sigma}}(t, \mathbf{z}(0))=0$ for all $t \geq 0$, then it means that the effect of $\bar{x}(0)$ is not appearing in the output measurements. Hence, in that case, $\boldsymbol{\Sigma}$ is not average observable. In the following, we provide a necessary condition for average observability. For the proof, please refer to Appendix.

Theorem 1. Let Assumption 1 hold. Then, $\boldsymbol{\Sigma}$ is average observable only if

$$
\operatorname{rank}\left[\begin{array}{l}
F \\
\mathbf{p}^{T}
\end{array}\right]=\operatorname{rank} F
$$

where $F \in \mathbb{R}^{n_{1} \times n_{2}}$ is given in (5) and $\bar{x}(t)=\mathbf{p}^{T} \mathbf{x}_{2}(t)$ is the average state with $\mathbf{x}_{2}(t)$ the state vector of unmeasured nodes and $\mathbf{p}=n_{2}^{-\frac{1}{2}} \mathbf{1}_{n_{2}}$.

Notice that the matrix $F$ contains two sets of information about $\Sigma$ : (i) The inflow configurations from unmeasured nodes $\mathcal{V}_{2}$ to gateway nodes $\mathcal{V}_{1}$ described by the matrix $A_{12}$ and (ii) the aggregated internal structure of subsystem formed by unmeasured nodes which is described by $\mathbf{p}^{T} A_{22}$. Here, the vector $\mathbf{p}$ can be considered as an aggregation vector. Hence, (7) requires that $\mathbf{p}$ lies in the rowspace of $F$. In the following, we provide a necessary and sufficient condition for average observability which is contingent on (7) - see Appendix for the proof.

Proposition 1. If (7) holds, then there exists a matrix $N=\left[\begin{array}{lll}\mathbf{n}_{1} & \ldots & \mathbf{n}_{\ell}\end{array}\right] \in \mathbb{R}^{\ell \times \ell}$ such that $N F=\mathbf{f} \mathbf{p}^{T}$, where $\mathbf{f} \in \mathbb{R}^{\ell}$ and $\ell=n_{1}+1$. Then, $\boldsymbol{\Sigma}$ is average observable if and only if $s \mathbf{n}_{\ell}-\mathbf{f} \neq 0$ for all $s \in \mathbb{R}$. 
The proposition that (7) implies the existence of $N \in \mathbb{R}^{\ell \times \ell}$ such that $N F=\mathbf{f} \mathbf{p}^{T}$ is straightforward. It is because (7) implies that $\mathbf{p}^{T}$ lies in the rowspace of $F$, therefore one can find a matrix $N$ that performs suitable $\ell$ row operations on $F$ to obtain $\mathbf{f} \mathbf{p}^{T}$. Then, the necessary and sufficient condition for average observability is that the last column of the matrix $N$ is linearly independent from the vector $\mathbf{f} \in \mathbb{R}^{\ell}$.

Theorem 2. The following statements hold:

(i) $\boldsymbol{\Sigma}$ is average observable if

$$
\operatorname{rank}\left[\begin{array}{c}
A_{12} \\
\mathbf{p}^{T}
\end{array}\right]=\operatorname{rank} A_{12} ;
$$

(ii) $\boldsymbol{\Sigma}$ is average observable if

$$
\operatorname{rank} F=\operatorname{rank} A_{12}=n_{1}
$$

where $A_{12}$ is given in (3), $F$ in (5), $n_{1}$ is the number of gateway nodes, and $\mathbf{p}=n_{2}^{-\frac{1}{2}} \mathbf{1}_{n_{2}}$ with $n_{2}$ the number of unmeasured nodes.

The sufficient condition (8) requires $\mathbf{p}^{T}$ to be in the rowspace of $A_{12}$, which is a matrix that contains the inflow configurations of gateway nodes from unmeasured nodes. To satisfy such a condition, it is necessary that every unmeasured node is connected to at least one gateway node. Precisely, for every $j \in \mathcal{V}_{2}$ there must exist $(i, j) \in \mathcal{E}$ with $i \in \mathcal{V}_{1}$.

On the other hand, the sufficient condition (9) requires $\mathbf{p}^{T} A_{22}$ to be in the rowspace of $A_{12}$. However sufficient for average observability, these conditions are difficult to satisfy for general networks. Nevertheless, they are easily computable for large-scale networks. The strictness of these conditions is a price to pay for reduced complexity.

Example 1. In Figure 2, where $\mathcal{V}_{1}$ are shown as black and $\mathcal{V}_{2}$ as green, we illustrate the sufficient condition (8). The edge weights are assumed to be 1 . Notice that the network depicted in Figure 2 is not observable since $\operatorname{rank} \mathcal{O}_{C, A}=$ $8<12$, where $C=\left[\begin{array}{ll}I_{2} & 0\end{array}\right]$. We have

$$
A_{12}=\left[\begin{array}{llllllllll}
1 & 1 & 1 & 1 & 1 & 0 & 0 & 0 & 0 & 0 \\
0 & 0 & 0 & 0 & 0 & 1 & 1 & 1 & 1 & 1
\end{array}\right],
$$

therefore (8) is satisfied and the network system is average observable.

Remark 2. The notion of average observability is a special case of functional observability, where the vector $\mathbf{p} \in \mathbb{R}^{n_{2}}$ represents any arbitrary linear combination of states. The necessary and sufficient condition of functional observability [10], [11] is given by

$$
\operatorname{rank}\left[\begin{array}{c}
\mathcal{O}_{C, A} \\
\mathcal{O}_{\mathbf{q}^{T}, A}
\end{array}\right]=\operatorname{rank} \mathcal{O}_{C, A},
$$

where $\mathcal{O}_{C, A}$ is given in (2) and $\mathcal{O}_{\mathbf{q}^{T}, A}$ can be obtained by replacing $C$ with $\mathbf{q}^{T}$ in (2) with $\mathbf{q}=\left[\begin{array}{ll}0^{T} & \mathbf{p}^{T}\end{array}\right]^{T} \in \mathbb{R}^{n}$. However, to verify this rank condition for large-scale network systems is computationally difficult. Therefore, to obtain tractable network topological conditions for functional observability, the approach presented in this paper can be easily generalized.

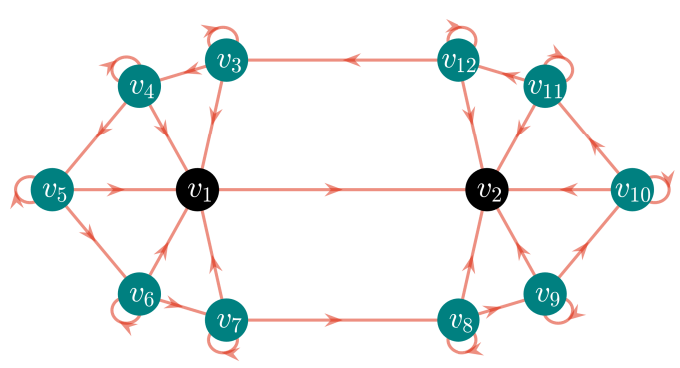

Fig. 2: An average observable network system

\section{AVERAGE DETECTABILITY OF NETWORK SYSTEMS}

If the network system is not average observable, then we cannot reconstruct the average state. However, the average state may be estimated by an observer if the network system is average detectable. The design of such an observer is spared for future research. We define the notion of average detectability as:

Definition 2. Suppose $\mathbf{u}(t)=0$ in $\boldsymbol{\Sigma}$. Let $\bar{x}(t)=\mathbf{p}^{T} \mathbf{x}_{2}(t)$ with $\mathbf{p}=n_{2}^{-\frac{1}{2}} \mathbf{1}_{n_{2}}$. Then, $\boldsymbol{\Sigma}$ is said to be average detectable if for all initial conditions $\mathbf{z}(0)=\left[\begin{array}{ll}\mathbf{x}_{1}^{T}(0) & \bar{x}(0)\end{array}\right]^{T} \in \mathbb{R}^{n_{1}+1}$ and the deviation vector $\boldsymbol{\sigma}(t) \in \mathbb{R}^{n_{2}}$ is such that $\mathbf{p}^{T} \boldsymbol{\sigma}(t)=0$ for all $t \geq 0$, it holds that the output $\mathbf{y}_{\boldsymbol{\sigma}}(t, \mathbf{z}(0))=\mathbf{x}_{1}(t)=0$ for all $t \geq 0$ implies $\bar{x}(t) \rightarrow 0$ as $t \rightarrow \infty$, where $\mathbf{y}_{\boldsymbol{\sigma}}(t, \mathbf{z}(0))$ is given by (6).

The above definition requires the unforced dynamics of $\bar{x}(t)$ to be stable for average detectability of $\boldsymbol{\Sigma}$. In the following, we present relatively mild sufficient conditions for average detectability, see Appendix for a proof.

Proposition 2. $\boldsymbol{\Sigma}$ is average detectable if and only if

(i) $\operatorname{rank} F \leq n_{1}$,

(ii) $\mathbf{p}^{T} A_{22} \mathbf{p}<0$,

where $F$ is given in (5), $A_{22}$ in (3), and $\mathbf{p}=n_{2}^{-\frac{1}{2}} \mathbf{1}_{n_{2}}$.

The intuitive explanation of rank deficiency of $F$ is that it allows us to cancel the effect of $\boldsymbol{\sigma}$ on the dynamics of the system $\boldsymbol{\Sigma}_{P}$. The matrix $F$, from (5), is such that it can be row-rank deficient in one of the following cases:

(C1) $\operatorname{rank} A_{12}<n_{1}$,

(C2) $\operatorname{rank} F=\operatorname{rank} A_{12}$.

Since $A_{12}$ contains the configuration of the inflows from $\mathcal{V}_{2}$ (unmeasured nodes) to $\mathcal{V}_{1}$ (gateway nodes), (C1) is satisfied if there exists a gateway node whose inflow configurations with respect to unmeasured nodes are linearly dependent on the inflow configurations of the other gateway nodes. This linear dependence is illustrated in Figure 3 , where $\mathcal{V}_{1}$ are shown as black, $\mathcal{V}_{2}$ as green, and the inflows from $\mathcal{V}_{2}$ to $\mathcal{V}_{1}$ are shown as blue edges. Note that inflow to both gateway nodes $v_{1}, v_{2} \in \mathcal{V}_{1}$ from the unmeasured node $v_{6} \in \mathcal{V}_{2}$ creates rank deficiency in $A_{12}$. 


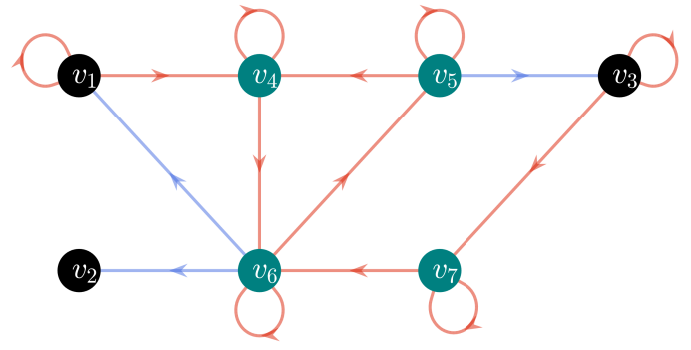

Fig. 3: An average detectable network system

Example 2. Consider a linear multi-compartmental system where the nodes represent the compartments. Each compartment or node $v_{i}$ contains a quantity $x_{i}(t)$ which satisfies

$$
\dot{x}_{i}(t)=\sum_{j \in \mathcal{N}_{i}^{\text {in }}} w_{i j} x_{j}(t)-\sum_{k \in \mathcal{N}_{i}^{\text {out }}} w_{k i} x_{i}(t),
$$

where the first term in the right hand side is flow in to node $v_{i}$ and the second term is flow out from node $v_{i}$. The compartments share their quantities with their out-neighbors and the edge weights $w_{i j}$ act as amplification parameters. Let the input $\mathbf{u}(t)=0$, then the system matrices $A$ and $C$ of the network system shown in Figure 3 are:

$$
\begin{aligned}
A & =\left[\begin{array}{rrr|rrrr}
-4 & 0 & 0 & 0 & 0 & 3 & 0 \\
0 & 0 & 0 & 0 & 0 & 5 & 0 \\
0 & 0 & -5 & 0 & 4 & 0 & 0 \\
\hline 4 & 0 & 0 & -3 & 2 & 0 & 0 \\
0 & 0 & 0 & 0 & -6 & 4 & 0 \\
0 & 0 & 0 & 3 & 0 & -12 & 1 \\
0 & 0 & 5 & 0 & 0 & 0 & -1
\end{array}\right] \\
C & =\left[\begin{array}{rrr|rrrr}
1 & 0 & 0 & 0 & 0 & 0 & 0 \\
0 & 1 & 0 & 0 & 0 & 0 & 0 \\
0 & 0 & 1 & 0 & 0 & 0 & 0
\end{array}\right],
\end{aligned}
$$

where the matrix partitions depict the partitions in (3) and the black nodes $v_{1}, v_{2}$, and $v_{3}$ are the gateway nodes. The network system is not observable since the observability rank condition is not satisfied, i.e., $\operatorname{rank} \mathcal{O}_{C, A}=6$. Also, it is not average observable since it doesn't satisfy (7). In the following, we check for the conditions of average detectability.

First, (C1) is satisfied with $\operatorname{rank} A_{12}=2<n_{1}=3$. Second, $\mathbf{p}=\frac{1}{2}\left[\begin{array}{llll}1 & 1 & 1 & 1\end{array}\right]^{T}$ and $\mathbf{p}^{T} A_{22}=\left[\begin{array}{llll}0 & -2 & -4 & 0\end{array}\right]$, which lies in the span of the rows of $A_{12}$. Hence, the condition $\operatorname{rank} F \leq n_{1}$ is satisfied. Moreover, Proposition 2(ii) is satisfied since $\mathbf{p}^{T} A_{22} \mathbf{p}=-3<0$. Therefore, the network system in Figure 3(b) is average detectable.

Theorem 3. $\Sigma$ is average detectable if

$$
\mathbf{p}^{T} A_{22}=-\gamma \mathbf{p}^{T},
$$

where $\gamma>0, A_{22}$ is given in (3), and $\mathbf{p}=n_{2}^{-\frac{1}{2}} \mathbf{1}_{n_{2}}$.

Proof. Suppose (10) holds, then $\mathbf{p}^{T} A_{22} \mathbf{p}=-\gamma<0$. Moreover, it also holds that $F \boldsymbol{\sigma}(t)=\hat{F} \boldsymbol{\sigma}(t)$, where
$\hat{F}=\left[\begin{array}{c}A_{12} \\ 0\end{array}\right]$ and $F$ is given in (5). It is because $\mathbf{p}^{T} A_{22} \boldsymbol{\sigma}(t)=-\gamma \mathbf{p}^{T} \boldsymbol{\sigma}(t)=0$. Therefore, both conditions of Proposition 2 are satisfied.

\section{CONCLUding REMARKS}

Large-scale network systems are often unobservable with dedicated state measurements at few gateway nodes. Therefore, we resorted to the problem of reconstructing the average state of the unmeasured nodes, and defined the notions of average observability and average detectability. The complexity of the problem is reduced by obtaining the projected system with dynamics in lower dimensional state space. Referring to average observability as $\mathrm{AO}$ and average detectability as $\mathrm{AD}$, the results in this paper are summarized as:

(i) $\mathrm{AO} \Longrightarrow$ Theorem 1 .

(ii) $\mathrm{AO} \Longleftrightarrow$ Proposition 1 .

(iii) Theorem $2 \Longrightarrow \mathrm{AO}$.

(iv) $\mathrm{AD} \Longleftrightarrow$ Proposition 2 .

(v) Theorem $3 \Longrightarrow \mathrm{AD}$.

The future prospects include (a) the design of average state observer; (b) the extension of current framework to nonlinear network systems with multiple clusters of unmeasured nodes; and (c) reconstruction of a nonlinear functional of the state.

\section{ACKNOWLEDGEMENT}

This work is supported by European Research Council (ERC) under the European Union's Horizon 2020 research and innovation programme (ERC-AdG no. 694209, ScaleFreeBack, website: http://scale-freeback.eu/).

\section{APPENDIX}

\section{A. Proof of Lemma 1}

For the pair $(H, E)$ to be observable, the following $\mathrm{PBH}$ test is equivalent:

$$
\operatorname{rank}\left[\begin{array}{cc}
s I-A_{11} & -A_{12} \mathbf{p} \\
-\mathbf{p}^{T} A_{21} & s-\mathbf{p}^{T} A_{22} \mathbf{p} \\
I & 0
\end{array}\right]=n_{1}+1, \quad \forall s \in \mathbb{C} .
$$

Thus, it is clear that $A_{12} \mathbf{p} \neq 0$ is necessary and sufficient for the observability of the pair $(H, E)$. The matrix $A_{12}$ is nonnegative with $i j$-entry positive if there exists an edge $(i, j) \in \mathcal{E}$, where $i \in \mathcal{V}_{1}$ and $j \in \mathcal{V}_{2}$. Therefore, if there exists such an edge, we have $A_{12} \mathbf{p} \neq 0$.

\section{B. Proof of Theorem 1}

To reconstruct the average state $\bar{x}(t)$ from $\boldsymbol{\Sigma}_{P}$, it is necessary that the effect of $\boldsymbol{\sigma}(t)$ is canceled. Consider left multiplying the state equation of $\Sigma_{P}$ by $N \in \mathbb{R}^{\ell \times \ell}$, where $\ell=n_{1}+1$, which gives a descriptor system

$$
\begin{array}{ll}
N \dot{\mathbf{z}}(t) & =N E \mathbf{z}(t)+N F \boldsymbol{\sigma}(t)+N G \mathbf{u}(t) \\
\mathbf{y}(t) & =H \mathbf{z}(t) .
\end{array}
$$

Therefore, for observability of (11), it is necessary that $N F \boldsymbol{\sigma}(t)=0$. To prove that it is indeed equivalent to (7), note that $\boldsymbol{\sigma}(t)=\left(I-\mathbf{p} \mathbf{p}^{T}\right) \mathbf{x}_{2}(t)$, where the rank of $I-\mathbf{p} \mathbf{p}^{T}$ is equal to $n_{2}-1$ and its nullspace is spanned by $\mathbf{p}$. Since $\mathbf{x}_{2}(t) \in \mathbb{R}^{n_{2}}$ is arbitrary, we have $N F \boldsymbol{\sigma}(t)=0$ if and only if $N F=\mathbf{f} \mathbf{p}^{T}$, where $\mathbf{f} \in \mathbb{R}^{\ell}$, which is equivalent to (7). 


\section{Proof of Proposition 1}

Consider again a descriptor system (11) with $N F \boldsymbol{\sigma}(t)=0$ and the output $\mathbf{y}(t)=H \mathbf{z}(t)$. This system is observable, see [14], [15], if and only if

$$
\operatorname{rank}\left[\begin{array}{c}
s N-N E \\
H
\end{array}\right]=\ell, \quad \forall s \in \mathbb{C},
$$

where $\ell=n_{1}+1$. Since (7) holds, we have $N F=\mathbf{f} \mathbf{p}^{T}$, where $\mathbf{f} \in \mathbb{R}^{\ell}$. Let $N=\left[\begin{array}{lll}\mathbf{n}_{1} & \ldots & \mathbf{n}_{\ell}\end{array}\right]$, where $\mathbf{n}_{i} \in \mathbb{R}^{\ell}$ for $i=1, \ldots, \ell$. From (5), notice that $E=[* F \mathbf{p}]$, where $*$ denotes the terms which are trivial in the following proof. Hence, $N E=\left[\begin{array}{ll}* & \mathbf{f}\end{array}\right]$ and (12) is given as

$$
\operatorname{rank}\left[\begin{array}{cc}
* & s \mathbf{n}_{\ell}-\mathbf{f} \\
I_{n_{1}} & 0
\end{array}\right]=\ell, \quad \forall s \in \mathbb{C},
$$

which is equivalent to $s \mathbf{n}_{\ell}-\mathbf{f} \neq 0$ for all $s \in \mathbb{R}$ since $\mathbf{n}_{\ell}, \mathbf{f} \in \mathbb{R}^{\ell}$. In other words, if $\mathbf{n}_{\ell} \neq 0$, then $\mathbf{n}_{\ell}$ and $\mathbf{f}$ must be linearly independent.

\section{Proof of Theorem 2}

(i) Let $N=\left[\begin{array}{ll}N_{1} & 0\end{array}\right]$, i.e. $n_{\ell}=0$. Then, if (8) hold, then $N F=N_{1} A_{12}=\mathbf{f} \mathbf{p}^{T}$, where $\mathbf{f} \neq 0$. Therefore, the necessary and sufficient condition of average observability in Proposition 1 is satisfied.

(ii) Suppose rank $A_{12}=n_{1}$, then in the following we prove that $\mathbf{n}_{\ell}$ and $\mathbf{f}$ are linearly independent if and only if $\operatorname{rank} N \geq 2$ :

For the necessity, suppose $\operatorname{rank} N=1$ and $\mathbf{n}_{\ell}, \mathbf{f}$ are linearly independent. Then, $N=\left[\begin{array}{llll}\alpha_{1} \mathbf{m}_{1} & \alpha_{2} \mathbf{m}_{1} & \ldots & \alpha_{\ell} \mathbf{m}_{1}\end{array}\right]^{T}$, where $\alpha_{j} \mathbf{m}_{1}^{T}=\alpha_{j}\left[m_{1}^{1} \ldots m_{1}^{\ell}\right]$ is the $j$-th row of $N$ and $\alpha_{j}, m_{1}^{j}$ are scalars, for $j=1, \ldots \ell$. Then, we know that the last column of $N$ is given by $\mathbf{n}_{\ell}=m_{1}^{\ell}\left[\begin{array}{lll}\alpha_{1} & \ldots \alpha_{\ell}\end{array}\right]^{T}$. Let $\mathbf{m}_{1}^{T} F=f_{1} \mathbf{p}^{T}$, where $f_{1} \in \mathbb{R}$, since (7) holds, then $N F=\mathbf{f} \mathbf{p}^{T}$ and $\mathbf{f}=f_{1}\left[\begin{array}{lll}\alpha_{1} & \ldots & \alpha_{\ell}\end{array}\right]^{T}$. Hence, we arrive at a contradiction because $\mathbf{n}_{\ell}, \mathbf{f}$ are linearly dependent.

For the sufficiency, suppose $\operatorname{rank} N=2$ and let $N=$ $\left[\begin{array}{lll}\mathbf{m}_{1} & \mathbf{m}_{2} & \ldots\end{array}\right]^{T}$, where $\mathbf{m}_{1}^{T}$ and $\mathbf{m}_{2}^{T}$ are linearly independent rows and ... represent the rows which are in $\operatorname{span}\left\{\mathbf{m}_{1}, \mathbf{m}_{2}\right\}$. Let $\mathbf{s}=\left[\begin{array}{lllll}s_{1} & s_{2} & 0 & \ldots & 0\end{array}\right]^{T}$ be such that atleast one of $s_{1}, s_{2}$ is nonzero and $\mathbf{s}^{T} \mathbf{n}_{\ell}=0$. Let $\hat{N}=\left[\begin{array}{lll}\mathbf{n}_{1} & \ldots & \mathbf{n}_{\ell-1}\end{array}\right]$, then $\hat{N} A_{12}+\mathbf{n}_{\ell} \mathbf{p}^{T} A_{22}=\mathbf{f} \mathbf{p}^{T}$. Multiplying $\mathbf{s}^{T}$ from left gives $\mathbf{s}^{T} \hat{N} A_{12}=\mathbf{s}^{T} \mathbf{f} \mathbf{p}^{T}$. For $\mathbf{n}_{\ell}$ and $\mathbf{f}$ to be linearly dependent, it must hold that $\mathbf{s}^{T} \mathbf{f}=0$. This implies $\mathbf{s}^{T} \hat{N}=0$ since rank $A_{12}=n_{1}$, i.e., full row rank. But $\mathbf{s}^{T} N=\mathbf{s}^{T}\left[\hat{N} \mathbf{n}_{\ell}\right] \neq 0$, because the first two rows of $N$ are linearly independent. This proves the sufficiency that if $\operatorname{rank} N \geq 2$, then $\mathbf{n}_{\ell}$, $\mathbf{f}$ are linearly independent.

Finally, we prove that $\operatorname{rank} N \geq 2$ implies $\operatorname{rank} F \leq n_{1}$. From (7), $\operatorname{rank} N F=1$. If $\operatorname{rank} F=\ell$, then $\operatorname{rank} N F=$ $\operatorname{rank} N \neq 1$. Therefore, $\operatorname{rank} F<\ell$, where $\ell=n_{1}+1$. But rank $A_{12}=n_{1}$, hence $\operatorname{rank} F=n_{1}$ from (5).

\section{E. Proof of Proposition 2}

First, consider (11) with $N F=0$. Such an $N \in \mathbb{R}^{\ell \times \ell}$, $\ell=n_{1}+1$, exists if and only if Proposition 2(i) holds. Second, we know that $\boldsymbol{\sigma}(t)=\left(I-\mathbf{p p}^{T}\right) \mathbf{x}_{2}(t)$. But, $(I-$ $\left.\mathbf{p} \mathbf{p}^{T}\right)$ is an idempotent matrix, i.e., $\left(I-\mathbf{p} \mathbf{p}^{T}\right)^{2}=\left(I-\mathbf{p} \mathbf{p}^{T}\right)$.
Therefore, we can write $F \boldsymbol{\sigma}(t)=F\left(I-\mathbf{p p}^{T}\right) \boldsymbol{\sigma}(t)$. Using these identities, the term $F \boldsymbol{\sigma}(t)$ in $\boldsymbol{\Sigma}_{P}$ can be replaced by $\hat{F} \boldsymbol{\sigma}(t)$, where

$$
\hat{F}=\left[\begin{array}{c}
A_{12} \\
\mathbf{p}^{T} \Delta_{22}
\end{array}\right]
$$

with $\Delta_{22}=\mathbf{p p}^{T} A_{22}-A_{22} \mathbf{p p}^{T}$. Now, there exists $\hat{N} \in$ $\mathbb{R}^{\ell \times \ell}$ such that $\hat{N} \hat{F}=0$ if and only if $\operatorname{rank} \hat{F}<\ell$. Let $\hat{N}=\left[\begin{array}{ll}\hat{N}_{1} & \hat{N}_{2}\end{array}\right]$, then $\hat{N}_{1} A_{12}+\hat{N}_{2} \mathbf{p}^{T} \Delta_{22}=0$. Since $\mathbf{p}^{T} \Delta_{22} \mathbf{p}=0$, we have $\hat{N}_{1} A_{12} \mathbf{p}=0$. Then, from (12)

$$
\operatorname{rank}\left[\begin{array}{c}
s \hat{N}-\hat{N} E \\
H
\end{array}\right]=\operatorname{rank}\left[\begin{array}{cc}
* & \hat{N}_{2}\left(s-\mathbf{p}^{T} A_{22} \mathbf{p}\right) \\
I_{n_{1}} & 0
\end{array}\right],
$$

where ' $*$ ' is an irrelevant term in the rank of the matrix. It can be easily seen that (12) doesn't hold at $s=\mathbf{p}^{T} A_{22} \mathbf{p}$. But if $\mathbf{p}^{T} A_{22} \mathbf{p}<0$, then the above rank condition holds for all $s \in \mathbb{C}_{\geq 0}$ and, hence, $\boldsymbol{\Sigma}$ is average detectable.

\section{REFERENCES}

[1] M. Ji and M. Egerstedt, "Observability and estimation in distributed sensor networks," in 2007 46th IEEE Conference on Decision and Control. IEEE, 2007, pp. 4221-4226.

[2] A. Rahmani, M. Ji, M. Mesbahi, and M. Egerstedt, "Controllability of multi-agent systems from a graph-theoretic perspective," SIAM Journal on Control and Optimization, vol. 48, no. 1, pp. 162-186, 2009.

[3] Y.-Y. Liu, J.-J. Slotine, and A.-L. Barabási, "Controllability of complex networks," Nature, vol. 473, no. 7346, pp. 167-173, 2011.

[4] G. Notarstefano and G. Parlangeli, "Controllability and observability of grid graphs via reduction and symmetries," IEEE Transactions on Automatic Control, vol. 58, no. 7, pp. 1719-1731, 2013.

[5] A. Y. Kibangou and C. Commault, "Observability in connected strongly regular graphs and distance regular graphs," IEEE Transactions on Control of Network Systems, vol. 1, no. 4, pp. 360-369, 2014

[6] N. Monshizadeh, S. Zhang, and M. K. Camlibel, "Zero forcing sets and controllability of dynamical systems defined on graphs," IEEE Transactions on Automatic Control, vol. 59, no. 9, pp. 2562-2567, 2014

[7] T. Kailath, Linear systems. Prentice-Hall Englewood Cliffs, NJ, 1980.

[8] A. Rantzer and M. E. Valcher, "A tutorial on positive systems and large scale control," in 2018 IEEE Conference on Decision and Control (CDC). IEEE, 2018, pp. 3686-3697.

[9] J. P. Hespanha, D. Liberzon, D. Angeli, and E. D. Sontag, "Nonlinear norm-observability notions and stability of switched systems," IEEE Transactions on Automatic Control, vol. 50, no. 2, pp. 154-168, 2005.

[10] T. L. Fernando, H. M. Trinh, and L. Jennings, "Functional observability and the design of minimum order linear functional observers," IEEE Transactions on Automatic Control, vol. 55, no. 5, pp. 12681273,2010

[11] F. Rotella and I. Zambettakis, "A note on functional observability," IEEE Transactions on Automatic Control, vol. 61, no. 10, pp. 31973202,2016

[12] T. Ishizaki, K. Kashima, J.-i. Imura, and K. Aihara, "Model reduction and clusterization of large-scale bidirectional networks," IEEE Transactions on Automatic Control, vol. 59, no. 1, pp. 48-63, 2014.

[13] H. L. Trentelman, A. A. Stoorvogel, and M. Hautus, Control theory for linear systems. Springer-Verlag London, 2001.

[14] E. Yip and R. Sincovec, "Solvability, controllability, and observability of continuous descriptor systems," IEEE transactions on Automatic Control, vol. 26, no. 3, pp. 702-707, 1981.

[15] D. Cobb, "Controllability, observability, and duality in singular systems," IEEE transactions on Automatic Control, vol. 29, no. 12, pp. 1076-1082, 1984 